\title{
Functional independence, access to kidney transplantation and waitlist mortality
}

\author{
Nadia M. Chu ${ }^{1,2, \star}$, Stephanie Sison ${ }^{3, *}$, Abimereki D. Muzaale ${ }^{1, \star}$, Christine E. Haugen ${ }^{1}$, \\ Jacqueline M. Garonzik-Wang ${ }^{1}$, Daniel C. Brennan ${ }^{1}$, Silas P. Norman ${ }^{4}$, Dorry L. Segev ${ }^{1,2}$ and \\ Mara McAdams-DeMarco (D) ${ }^{1,2}$ \\ ${ }^{1}$ Department of Surgery, Johns Hopkins University School of Medicine, Baltimore, MD, USA, ${ }^{2}$ Department of Epidemiology, Johns Hopkins \\ Bloomberg School of Public Health, Baltimore, MD, USA, ${ }^{3}$ Department of Medicine, Johns Hopkins University School of Medicine, Baltimore, \\ MD, USA and ${ }^{4}$ Department of Medicine, University of Michigan School of Medicine, Baltimore, MD, USA
}

Correspondence to: Mara McAdams-DeMarco; E-mail: mara@jhu.edu

${ }^{\star}$ These authors contributed equally to this work.

\section{ABSTRACT}

Background. Approximately half of the patients who progress to end-stage kidney disease (ESKD) and undergo dialysis develop difficulties carrying out essential self-care activities, leading to institutionalization and mortality. It is unclear what percentage of kidney transplant (KT) candidates, a group of ESKD patients selected to be healthy enough to withstand transplantation, are functionally independent and whether independence is associated with better access to KT and reduced waitlist mortality.

Methods. We studied a prospective cohort of 3168 ESKD participants (January 2009 to June 2018) who self-reported functional independence in more basic self-care Activities of Daily Living (ADL) (needing help with eating, dressing, walking, grooming, toileting and bathing) and more complex instrumental ADL (IADL) (needing help using a phone, shopping, cooking, housework, washing, using transportation, managing medications and managing money). We estimated adjusted associations between functional independence (separately) and listing (Cox), waitlist mortality (competing risks) and transplant rates (Poisson).

Results. At KT evaluation, 92.4\% were independent in ADLs, but only $68.5 \%$ were independent in IADLs. Functionally independent participants had a higher chance of listing for KT [ADL: adjusted hazard ratio $(\mathrm{aHR})=1.55,95 \%$ confidence interval (CI) 1.30-1.87; IADL: $\mathrm{aHR}=1.39$, 95\% CI 1.26-1.52]. Among KT candidates, ADL independence was associated with lower waitlist mortality risk [adjusted subdistribution HR $(\mathrm{aSHR})=0.66,95 \%$ CI $0.44-0.98]$ and higher rate of $\mathrm{KT}$ [adjusted incidence rate ratio $(\mathrm{aIRR})=1.58,95 \% \mathrm{CI} 1.12-2.22]$; the same was not observed for IADL independence $(\mathrm{aSHR}=0.86$, 95\% CI 0.65-1.12; aIRR = 1.01, 95\% CI 0.97-1.19).

Conclusions. Functional independence in more basic self-care ADL was associated with better KT access and lower waitlist mortality. Nephrologists, geriatricians and transplant surgeons should screen KT candidates for ADLs, and identify interventions to promote independence and improve waitlist outcomes.

Keywords: functional performance, outcomes, renal

\section{INTRODUCTION}

Functional independence reflects a patient's ability to care for themselves $[1,2]$ and is a health priority for patients with advanced chronic kidney disease [3]. Lack of functional independence leads to poor outcomes including institutionalization in assisted care facilities [4] and mortality [5-7] in end-stage kidney disease (ESKD) patients of all ages. Dialysis initiation is associated with the transition from independence to dependence, regardless of the patient's age [7-9], resulting in 50\% of dialysis patients being functionally dependent [10-12]. Nearly one in five patients report needing help with at least one or more basic self-care activities of daily living (ADL) [13], including eating, dressing, walking, grooming, toileting and bathing [12, 14]. Furthermore, $\sim 80 \%$ of ESKD patients report needing help with more complex instrumental ADL (IADL) [15], such as using a phone, shopping, cooking, housework, washing, using transportation, managing medications and managing money [7, 9, $10,12,14,16-18]$. While it is likely that some kidney transplant (KT) candidates are not functionally independent resulting from their high burden of frailty and years of chronic conditions [19-21], the prevalence of functional independence in this population of ESKD patients is unknown.

While a lack of functional independence is associated with higher mortality risk in dialysis patients of all ages $[5,6,19]$, it remains unclear if the same holds true for those listed for KT. The risks associated with not being independent among KT candidates are unclear and cannot be extrapolated from 
patients undergoing hemodialysis, because KT candidates are a group of ESKD patients who are selected to be healthy enough to withstand transplant surgery. KT candidates are typically younger and healthier than the general ESKD population [22], and include patients not on dialysis who are pursuing preemptive KT. Among KT candidates, understanding the impact of functional independence and waitlist mortality as well as access to KT is important and novel.

There are clear links between certain domains of independence and adverse outcomes. For example, the inability to manage medications is likely associated with access to KT because treatment and medication nonadherence contribute to adverse ESKD outcomes, and is considered prior to listing [23, 24]. While some domains of independence are directly observable by clinicians during KT evaluation (such as inability to walk), others may not be (such as needing help with housework). Therefore, understanding the role of these individual self-care domains among KT candidates is important for the care of KT candidates.

To better understand the impact of functional independence on access to KT and waitlist mortality, we leveraged a prospective cohort study of ESKD participants undergoing evaluation for KT. The objectives of this study were to: (i) estimate the prevalence of functional independence overall and report the domains of independence in this population and (ii) test whether functional independence, overall and by domain, is associated with access to KT (including likelihood of listing and rates of $\mathrm{KT}$ ) and waitlist mortality. Understanding the role of functional independence in KT candidates may provide quantitative evidence that substantiates patient perspectives of an important health priority.

\section{MATERIALS AND METHODS}

\section{Participants}

We studied a prospective cohort of 3168 ESKD participants being evaluated for KT at Johns Hopkins University in Baltimore, Maryland (January 2009 to June 2018, $n=2910$ ) and the University of Michigan in Ann Arbor, Michigan (January 2015 to April 2016, $n=258$ ), as previously described [25-29]. Participants were included in the study if they were English-speaking, $\geq 18$ years of age and were measured for functional independence at the time of evaluation, as described below. Other participant characteristics were obtained via selfreport at KT evaluation or abstracted from medical records, including age, sex, race, education, body mass index (BMI), diabetes status, depressive symptoms (Center for Epidemiologic Studies Depression Scale score $<16$ ), bodily pain (based on the Kidney Disease Quality of Life), years on dialysis, cause of ESKD and Charlson comorbidity index (CCI) adapted for ESKD $[30,31]$. Surveys were verbally administered in English.

\section{Functional independence}

Functional independence was derived from the National Long Term Care Survey verbally administered by study staff at the time of evaluation for KT. Participants self-reported whether they needed help with eating, dressing, walking, grooming, toileting or bathing, which comprised the six basic self-care ADL domains [13]. Participants also self-reported whether they needed help with using a phone, shopping, cooking, housework, washing, using transportation, managing medications or managing money, which comprised the eight more complex self-care IADL domains [15]. An individual was classified as ADL independent if they were not functionally dependent in any of the ADL domains reported; we used a similar approach to define IADL independence. A Venn diagram was generated to depict the proportion of participants classified as both ADL and IADL independent. The physicians at Johns Hopkins Hospital and the University of Michigan were not aware of the functional independence assessment results at the listing meeting in which physicians decide whether or not to list a patient.

\section{Correlates of functional independence}

Among ESKD participants who were listed for KT (KT candidates) $(n=1703)$, odds ratios (ORs) for assessing associations with ADL independence and demographic and health characteristics were obtained from adjusted logistic regression models. The same methods were used to identify correlates of IADL independence.

\section{Chance of listing by functional independence}

Among ESKD participants being evaluated for KT $(n=3168)$, adjusted Cox proportional hazards regressions were used to estimate the chance of listing by ADL independence. Time to listing was defined as the time from evaluation for KT to the date of active placement on the KT waitlist or administrative censoring at the end of the study period (May 2018). Proportional hazards assumptions were confirmed by visual inspection of the complementary log-log plots and Schoenfeld residuals. All models were adjusting for age, sex, race, education and diabetes. To test whether the association between ADL independence and time to KT listing varied by participant age, sex and race, an interaction between participant age (age $<65$ versus age $\geq 65$ years), race (black versus non-black) or sex (male versus female) and ADL independence in separate models was explored using Wald tests. The impact of each of the six individual $\mathrm{ADL}$ domains on the chance of listing was subsequently explored in a single model; this model quantified the association for each individual ADL domain above and beyond the other domains. The same methods were used to assess the chance of listing by IADL independence, as well as the eight IADL domains.

\section{Waitlist mortality}

Among KT candidates ( $n=1703$ ), Fine and Gray competing risk models [32] were used to estimate the risk [subdistribution hazard ratios (SHR)] of waitlist mortality by ADL independence, accounting for KT as a competing risk. The time of origin was the date of active listing, and KT candidates were followed to date of death; candidates were censored at the end of the study period (May 2018) if it preceded mortality or KT. All models were adjusted for age, sex, race, education and diabetes. To test whether the association between ADL 
independence and mortality varied by participant age, sex and race, an interaction between participant age (age $<65$ versus age $\geq 65$ years), race (black versus non-black) or sex (female versus male) and ADL independence in separate models was explored using Wald tests. The impact of each of the six individual ADL domains on waitlist mortality was subsequently explored in a single model; this model quantified the association for each individual ADL domain above and beyond the other domains. The same methods were used to assess the chance of listing by IADL independence, as well as the eight IADL domains.

\section{Transplant rate}

Among KT candidates $(n=1703)$, Poisson regression was used to estimate adjusted incidence rate ratio (aIRR) by ADL independence, accounting for age, sex, race, education and diabetes. Person-years were calculated from the date of KT listing to the date of KT, mortality or censoring at the end of the study period (May 2018). To test whether the association between ADL independence and transplant rate varied by participant age, sex and race, an interaction between participant age (age $<65$ versus age $\geq 65$ years), race (black versus non-black) or sex (female versus male) and ADL independence in separate models was explored using Wald tests. The impact of each of the six individual ADL domains on transplant rate was subsequently explored in a single model; this model quantified the association for each individual ADL domain above and beyond the other domains. The same methods were used to assess the chance of listing by IADL independence, as well as the eight IADL domains.

\section{Sensitivity analysis}

We conducted a sensitivity analysis additionally adjusting for CCI, diagnosis of dementia and the Modified Mini-Mental State (3MS) score for global cognitive function to determine if the impact of ADL and IADL independence on waitlist outcomes remained robust to the presence of comorbidities and cognitive function. We additionally conducted sensitivity analyses adjusting for depressive symptoms and bodily pain to assess whether inferences could be explained by these often undertreated symptoms.

\section{Statistical analysis}

All analyses were performed using Stata version 14.0/MP for Linux (Stata Corp, College Station, TX, USA).

\section{RESULTS}

\section{Study population}

Among the 3168 ESKD participants at time of KT evaluation (Table 1 and Figure 1), the median [interquartile range (IQR)] age was 56.0 years (45.3-64.8), with ages ranging from 18 to 89 years; $24.7 \%$ were over the age of 65 years, $58.9 \%$ were male, $45.9 \%$ were black and $44.9 \%$ had no more than a high school education. Additionally, $41.9 \%$ had diabetes as comorbidity, and $21.4 \%$ had diabetes as the cause of ESKD. ESKD participants had been on dialysis for a median (IQR) of 1.4 years (0.6-
3.3) at the time of KT evaluation, and 52.3\% were undergoing hemodialysis.

\section{Functional independence at time of evaluation}

At the time of evaluation, $92.5 \%$ were independent in basic self-care ADLs, and $68.5 \%$ were independent in more complex IADLs (Table 1). Older ESKD participants had similar levels of ADL independence (94.8\% versus $95.6 \%$ ) but were less likely with have IADL independence (65.8\% versus $69.4 \%$ ) compared with their younger counterparts. The prevalence of ADL independence by domain ranged from $95.2 \%$ (walking) to $99.4 \%$ (eating). By comparison, the prevalence of IADL independence by domain ranged from $76.3 \%$ (shopping) to $84.4 \%$ (housekeeping). Among the ESKD participants, 66.4\% were classified as having both ADL and IADL independence (Figure 2).

Those with ADL or IADL independence were generally younger (ADL: median age $=55.7$ versus 58.7 years; IADL: median age $=55.5$ versus 57.0 years), and more likely to be male (ADL: $59.7 \%$ versus $50.2 \%$; IADL: $60.0 \%$ versus $57.0 \%$ ), white (ADL: $49.0 \%$ versus $46.0 \%$; IADL: $49.0 \%$ versus $48.0 \%$ ) and have higher educational attainment (ADL: $58.3 \%$ versus $52.3 \%$; IADL: $59.6 \%$ versus $53.8 \%$ ). Additionally, those with ADL or IADL independence were less likely to have diabetes as a comorbidity (ADL: $40.7 \%$ versus 56.5\%; IADL: $39.3 \%$ versus 47.5\%) and diabetes as a cause of ESKD (ADL: $20.6 \%$ versus $33.0 \%$; IADL: $19.8 \%$ versus $25.0 \%$ ).

\section{Correlates of functional independence at time of evaluation}

Among KT candidates $(n=1703)$, males had a 1.65 times [95\% confidence interval (CI) 1.09-2.52; $\mathrm{P}=0.02$ ] greater odds of having ADL independence, and for every $1-\mathrm{U}$ increase in the comorbidity index, KT candidates had a lower odds [adjusted OR $(\mathrm{aOR})=0.91,95 \%$ CI $0.83-0.99]$ of having ADL independence (Table 2). Similarly, for every 1-U increase in the comorbidity index, KT candidates had lower odds $(\mathrm{aOR}=0.88,95 \%$ CI $0.83-0.93$ ) of IADL independence. Additionally, for every $10-\mathrm{kg} / \mathrm{m}^{2}$ increase in BMI, KT candidates had 1.36 times $(95 \%$ CI 1.11-1.65; $\mathrm{P}<0.001)$ increased odds of having IADL independence.

\section{Chance of listing and functional independence}

ESKD participants $(n=3168)$ were followed for a median (IQR) of 0.4 years $(0.1-1.2)$ and a total of 2832 person-years, during which 1703 (53.8\%) were listed for KT. ADL independence was associated with 1.55 times (95\% CI 1.30-1.87; $\mathrm{P}<0.001$ ) higher chance of listing (Table 3). The association between ADL independence and a chance of listing did not differ by age $(P=0.29)$, sex $(P=0.86)$ or race $(P=0.39)$. Independence in each ADL domains, including dressing, walking, grooming, using a toilet and bathing, was associated with a higher chance of listing after adjusting for the other respective domains; independence in eating was the only domain that was not associated with a higher chance of listing after adjusting for the other respective domains [adjusted $\mathrm{HR}(\mathrm{aHR})=1.69,95 \%$ CI 0.91-3.15; $\mathrm{P}=0.10$ ] (Figure 3). 
Table 1. Characteristics of ESKD participants at time of evaluation for kidney transplantation $(n=3168)^{a}$

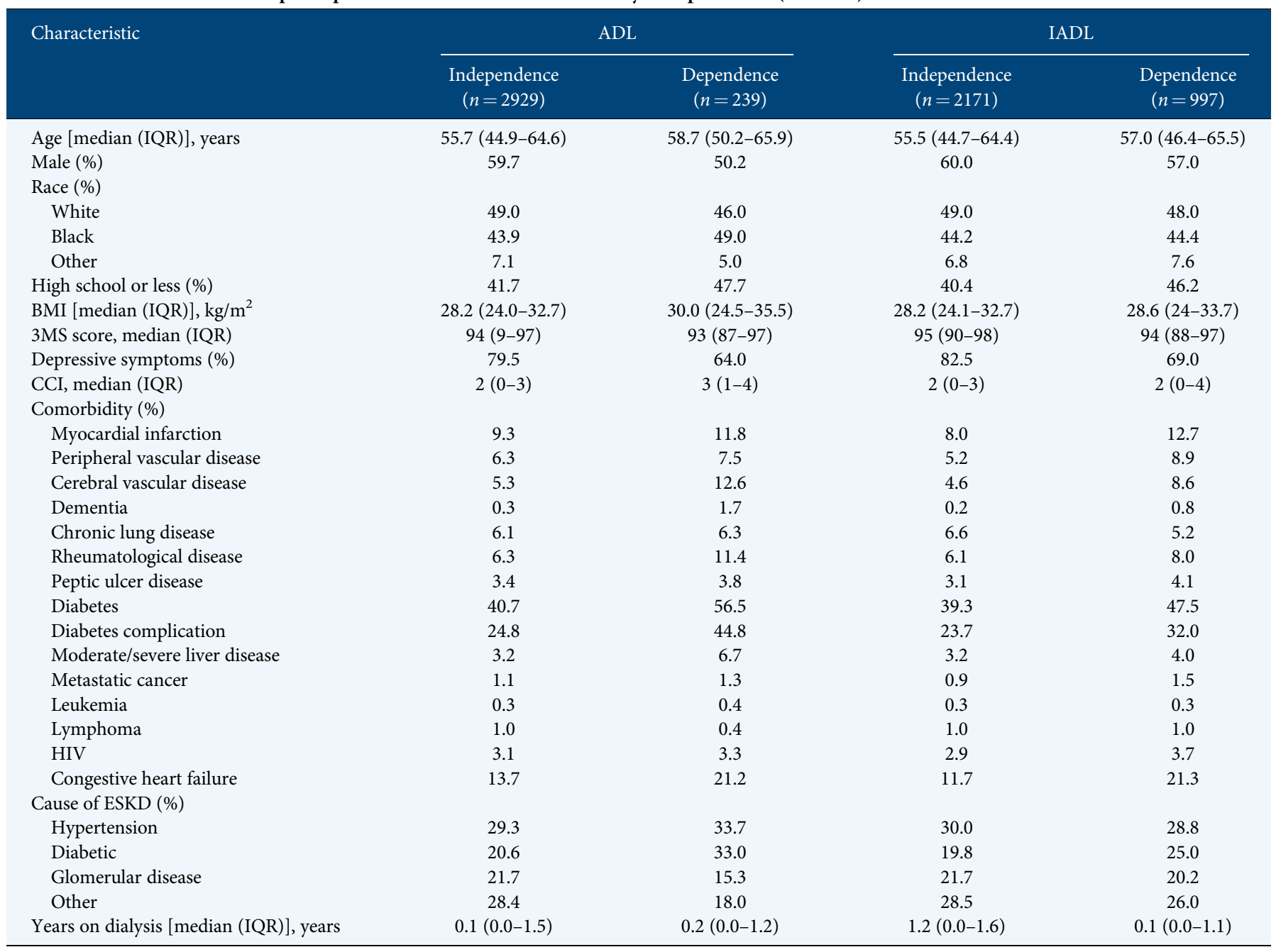

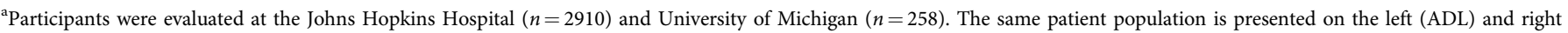
(IADL) hand sides of the Table. Depressive symptoms are characterized by a Center for Epidemiologic Studies Depression score of $<16$. The description of years on dialysis excludes those who were preemptively listed.

Similarly, IADL independence was associated with a higher chance of listing $(\mathrm{aHR}=1.39,95 \%$ CI $1.30-1.52 ; \mathrm{P}<0.001)$ (Table 3). The association between IADL independence and a chance of listing did not differ by age $(\mathrm{P}=0.85)$, sex $(\mathrm{P}=0.91)$ or race $(\mathrm{P}=0.37)$. Independence in each of the IADL domains, including using a phone, shopping, cooking, washing, using transportation, managing medications and managing money, was associated with a higher chance of listing after adjusting for the other respective domains; independence in housekeeping was the only domain that was not associated with a higher chance of listing after adjusting for the other respective domains $(\mathrm{aHR}=1.10,95 \% \mathrm{CI} 0.98-1.24 ; \mathrm{P}=0.11)$ (Figure 3 ).

\section{Waitlist mortality and functional independence}

There were $1703 \mathrm{KT}$ candidates who were listed for transplantation as active and they were followed for a median (IQR) of 1.2 years (0.5-2.5 years) and a total of 3103 person-years, during which 244 died before receiving KT. ADL independence was associated with a $34 \%(\mathrm{SHR}=0.66,95 \% \mathrm{CI} 0.44-0.98$; $\mathrm{P}=0.04$ ) lower risk of waitlist mortality (Table 3 ). The association between ADL independence and waitlist mortality did not

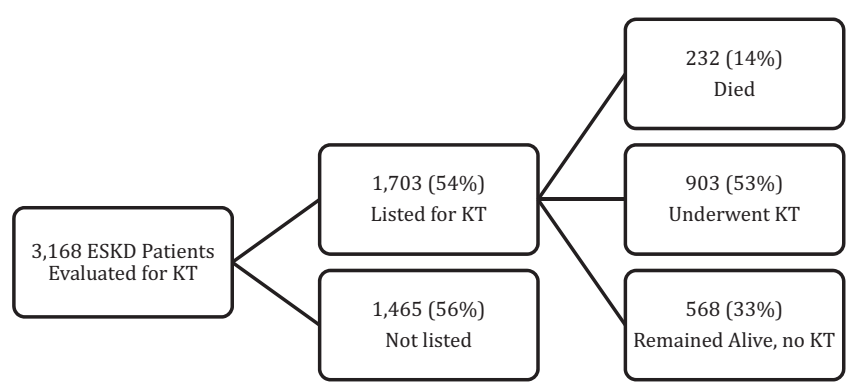

FIGURE 1: Proportion of those who died and underwent KT among ESKD participants ( $n=3168$, average follow-up time: 10.7 months) and KT candidates ( $n=1703$, average follow-up time: 2.4 years).

differ by age $(\mathrm{P}=0.54)$ or sex $(\mathrm{P}=0.09)$. However, the association between ADL independence and waitlist mortality differed by race $(\mathrm{P}=0.02)$, such that among blacks, $\mathrm{ADL}$ independence was not associated with waitlist mortality (SHR $=1.31,95 \%$ CI 0.50-3.20), while among non-blacks, ADL independence was associated with a $58 \%(\mathrm{SHR}=0.42,95 \%$ CI $0.26-0.68)$ lower 


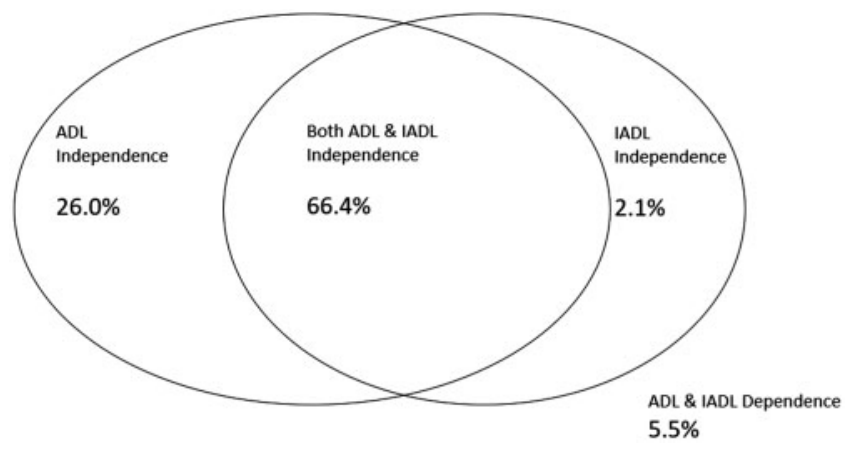

FIGURE 2: Proportion of ADL and IADL functional independence in ESKD participants being evaluated for kidney transplantation $(n=3168)$. Circles represent the proportion of participants evaluated for a kidney who are independent in ADLs and/or IADLs.

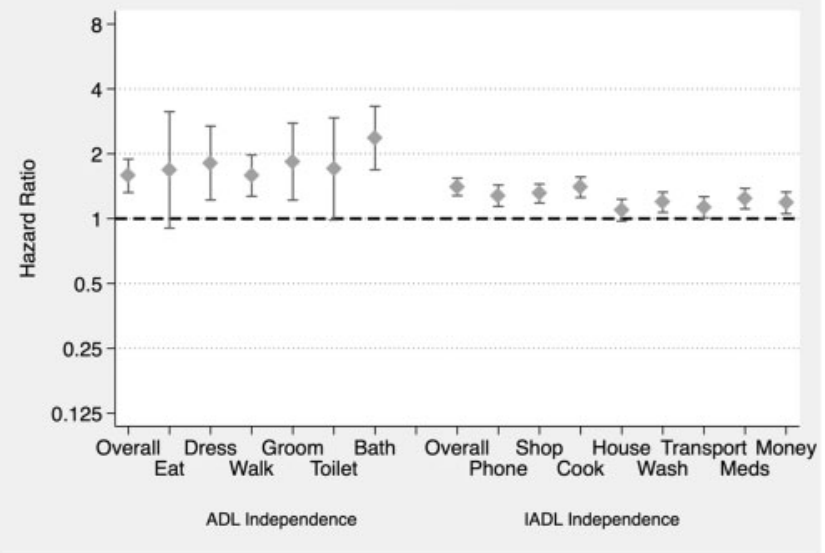

FIGURE 3: Chance of listing by domains of ADL and IADL functional independence among ESKD participants evaluated for kidney transplantation $(n=3168)$. Dots represent aHRs comparing the functionally independent with the functionally dependent in the specified daily activity; the bars represent the $95 \%$ CI of the HR. All models adjusted for age, sex, race, education and diabetes status. The $y$-axis is on a log scale.

risk of waitlist mortality. Independence in eating $(\mathrm{SHR}=0.32$, 95\% CI 0.15-0.70; $\mathrm{P}=0.01)$ and dressing $(\mathrm{SHR}=0.42,95 \% \mathrm{CI}$ $0.19-0.97 ; \mathrm{P}=0.04)$ were the only $\mathrm{ADL}$ domains associated with lower waitlist mortality after adjusting for the other respective domains (Figure 4).

IADL independence was not associated with waitlist mortality $(\mathrm{SHR}=0.86,95 \% \mathrm{CI} 0.65-1.12 ; \mathrm{P}=0.28$ ) (Table 3$)$, nor were any of its individual domains (Figure 4). Additionally, the association between IADL independence and waitlist mortality did not differ by age $(\mathrm{P}=0.54)$, sex $(\mathrm{P}=0.05)$ or race $(\mathrm{P}=0.33)$.

\section{Transplant rate by functional independence}

Of the $1703 \mathrm{KT}$ candidates, the median (IQR) follow-up time was 0.8 years ( $0.3-2.0$ years) with a total of 2317 personyears, during which 967 received KT. ADL independence was associated with a higher transplant rate $(\mathrm{IRR}=1.58,95 \% \mathrm{CI}$ $1.12-2.22 ; \mathrm{P}=0.01$ ) (Table 3 ). The association between $\mathrm{ADL}$

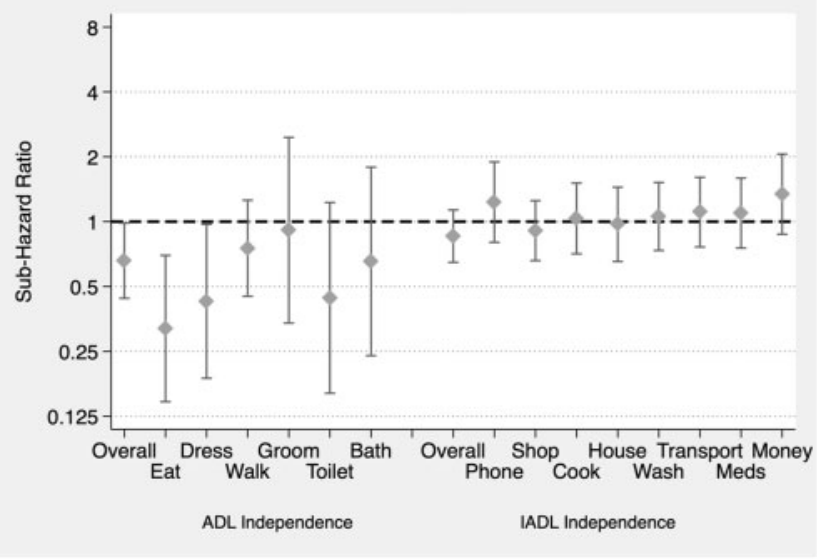

FIGURE 4: Risk of waitlist mortality by domains of functional independence (ADL/IADL) among KT candidates $(n=1703)$. Dots represent the aSHRs comparing those who were functionally independent with those who were functionally dependent in the specified daily activity; bars represent the $95 \%$ CI of the SHR. All models adjusted for age, sex, race, education and diabetes status. The $y$-axis is on a $\log$ scale.

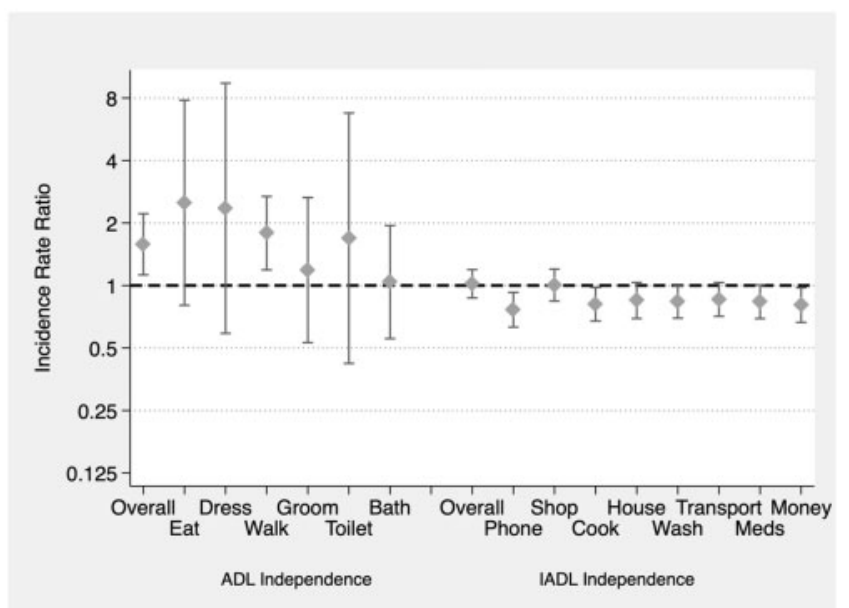

FIGURE 5: Rate of kidney transplantation by domains of functional independence (ADL/IADL) among KT candidates $(n=1703)$. Dots represent aIRRs comparing those who were functionally independent to those who were functionally dependent in the specified daily activity; bars represent the $95 \%$ CI of the IRRs. All models adjusted for age, sex, race, education and diabetes status. The $y$-axis is on a $\log$ scale.

independence and transplant rate did not differ by sex $(P=0.89)$ or race $(P=0.64)$. However, the association between $\mathrm{ADL}$ independence and transplant rate differed by age $(\mathrm{P}=0.04)$, such that among older ages, $\mathrm{ADL}$ independence was associated with 3.05 times (95\% CI 1.30-7.15) higher KT rate, while among younger ages, there was no association between $\mathrm{ADL}$ independence and $\mathrm{KT}$ rate $(\mathrm{IRR}=1.16$, 95\% CI 0.80 1.70). Of the ADL domains, only independence in walking was associated with a higher transplant rate after adjusting for the other respective domains $(\mathrm{IRR}=1.79,95 \%$ CI 1.19-2.69; $\mathrm{P}=0.005)$. Independence in eating, dressing, grooming, using a 
Table 2. Correlates of functional independence (ADL/IADL) among KT candidates $(n=1703)^{\mathrm{a}}$

\begin{tabular}{|c|c|c|c|c|}
\hline \multirow[t]{2}{*}{ Correlate } & \multicolumn{2}{|c|}{ ADL independence } & \multicolumn{2}{|c|}{ IADL independence } \\
\hline & $\mathrm{aOR}(95 \% \mathrm{CI})$ & P-value & $\mathrm{aOR}(95 \% \mathrm{CI})$ & P-value \\
\hline \multicolumn{5}{|l|}{ Age, years } \\
\hline $18-64$ & Reference & & Reference & \\
\hline$\geq 65$ & $0.91(0.56-1.48)$ & 0.71 & $0.94(0.72-1.22)$ & 0.63 \\
\hline Male & $1.65(1.09-2.52)$ & 0.02 & $1.22(0.97-1.52)$ & 0.08 \\
\hline Black race & $0.81(0.53-1.25)$ & 0.34 & $1.12(0.89-1.42)$ & 0.31 \\
\hline High school education or less & $0.83(0.54-1.25)$ & 0.38 & $0.88(0.70-1.11)$ & 0.29 \\
\hline BMI, per $10-\mathrm{kg} / \mathrm{m}^{2}$ increase & $0.96(0.67-1.37)$ & 0.83 & $1.36(1.11-1.65)$ & $<0.001$ \\
\hline Diabetes & $0.72(0.43-1.19)$ & 0.20 & $0.92(0.68-1.23)$ & 0.56 \\
\hline CCI, per unit increase & $0.91(0.83-0.99)$ & 0.04 & $0.88(0.83-0.93)$ & $<0.001$ \\
\hline Years on dialysis, per 10-year increase & $1.80(0.65-5.07)$ & 0.26 & $1.49(0.91-2.44)$ & 0.11 \\
\hline
\end{tabular}

${ }^{a}$ aORs for each characteristic were obtained from adjusted logistic regression models assessing associations with ADL independence simultaneously. In a separate model, the same approach was used to assess associations with IADL independence.

Table 3. Chance of listing, risk of waitlist mortality and KT rate ratios, by functional independence (ADL/IADL) ${ }^{a}$

\begin{tabular}{lcc} 
Outcome & Measure of association & P-value \\
\hline Chance of listing $(n=3168)$ & aHR $(95 \% \mathrm{CI})$ & \\
ADL independence & $1.55(1.30-1.87)$ & $<0.001$ \\
IADL independence & $1.39(1.26-1.52)$ & $<0.001$ \\
Risk of waitlist mortality $(n=1703)$ & aSHR $(95 \% \mathrm{CI})$ & \\
ADL independence & $0.66(0.44-0.98)$ & 0.04 \\
IADL independence & $0.86(0.65-1.12)$ & 0.28 \\
Transplant rate $(n=1703)$ & aIRR $(95 \% \mathrm{CI})$ & \\
ADL independence & $1.58(1.12-2.22)$ & 0.01 \\
IADL independence & $1.01(0.87-1.19)$ & 0.81 \\
\hline
\end{tabular}

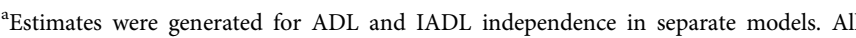
analyses were adjusted for age, sex, race, education and diabetes status.

toilet and bathing was not associated with the transplant rate (Figure 5).

IADL independence was not associated with the transplant rate $(\mathrm{IRR}=1.01,95 \% \mathrm{CI} 0.87-1.19 ; \mathrm{P}=0.81)$ (Table 3$)$. The association between IADL independence and transplant rate did not differ by age $(\mathrm{P}=0.83)$, sex $(\mathrm{P}=0.30)$ or race $(\mathrm{P}=0.97)$. However, several domains of IADL independence were associated with lower transplant rates after adjusting for the other respective domains, including independence in use of a phone $(\mathrm{IRR}=0.77, \quad 95 \% \quad$ CI $\quad 0.64-0.94 ; \quad \mathrm{P}=0.009), \quad$ cooking $(\mathrm{IRR}=0.82,95 \%$ CI $0.87-0.98 ; \mathrm{P}=0.03)$, washing $(\mathrm{IRR}=0.83$, $95 \%$ CI $0.69-0.99 ; \quad \mathrm{P}=0.04)$, and managing money $(\mathrm{IRR}=0.80,95 \% \mathrm{CI} 0.66-0.97 ; \mathrm{P}=0.02)$. Independence in shopping, using transportation, managing medications, managing money and housekeeping were not associated with transplant rate after adjusting for the other respective domains (Figure 5).

\section{Sensitivity analysis}

Across all sensitivity analyses, inferences remained robust. Specifically, after further adjusting all models for CCI, diagnosis of dementia and $3 \mathrm{MS}$ score, both ADL $(\mathrm{aHR}=1.54)$ and IADL $(\mathrm{aHR}=1.12)$, independence were associated with a greater chance of listing. Additionally, ADL independence was associated with a lower risk of waitlist mortality $(\mathrm{aSHR}=0.79)$ and an increased rate of transplant $(\mathrm{aIRR}=1.38)$. Results also remained robust after adjusting for depressive symptoms and bodily pain. For models controlling for depressive symptoms, both ADL $(\mathrm{aHR}=1.47)$ and IADL $(\mathrm{aHR}=1.12)$, independence were associated with a greater chance of listing, and ADL independence was associated with a lower risk of waitlist mortality $(\mathrm{aSHR}=0.79)$ and increased rate of transplant $(\mathrm{aIRR}=1.38)$. After adjusting for bodily pain, both $\mathrm{ADL}(\mathrm{aHR}=1.66)$ and IADL $(\mathrm{aHR}=1.12)$ independence were also associated with a greater chance of listing, and ADL independence was associated with a lower risk of waitlist mortality $(\mathrm{aSHR}=0.46)$ and increased rate of transplant $(\mathrm{aIRR}=2.24)$.

\section{DISCUSSION}

In this prospective cohort study of 3168 ESKD participants, 92.4\% were independent in basic self-care ADLs, but only $68.5 \%$ were independent in more complex self-care IADLs at the time of evaluation for KT. ESKD participants with ADL or IADL independence at time of evaluation for KT had a higher chance of listing for KT (ADL: aHR $=1.55,95 \%$ CI 1.30-1.87; IADL: $\mathrm{aHR}=1.39,95 \% \mathrm{CI} 1.26-1.52$ ), and these associations did not differ by age, sex or race. Unlike KT candidates with IADL independence at the time of listing, KT candidates with $\mathrm{ADL}$ independence had a lower waitlist mortality risk $(\mathrm{SHR}=0.66,95 \% \mathrm{CI} 0.44-0.98)$ overall and particularly among non-black candidates $(\mathrm{SHR}=0.42, \quad 95 \%$ CI $0.26-0.68)$. Additionally, ADL independence was associated with a higher rate of $\mathrm{KT}$ overall (IRR $=1.58,95 \% \mathrm{CI} 1.12-2.22)$, and particularly among older adults aged $\geq 65$ years (IRR $=3.05,95 \%$ CI 1.30-7.15).

This study extends these findings among dialysis patients to a novel population of ESKD patients of all ages who are being evaluated for KT, in which $92.4 \%$ were functionally independent based on ADLs and 68.5\% were functionally independent based on IADLs. Even among this subgroup of ESKD patients selected to be healthy enough to withstand transplantation, these findings support prior research conducted among dialysis patients. Specifically, prior studies have found that impairments in basic self-care ADLs, including eating, dressing, walking, grooming, toileting and bathing, are less common than impairments in more complex self-care IADLs, such as using a phone, 
shopping, cooking, housework, washing, using transportation, managing medications and managing money [11].

Additionally, to our knowledge, this is the first study to investigate whether functional independence is associated with better outcomes in ESKD participants being evaluated for KT and candidates listed for KT. Prior research has mainly focused on the role of dependence on poor adverse outcomes among patients on dialysis generally $[4-7,16]$, findings from which cannot be generalized to KT candidates selected to be healthy enough to withstand transplant surgery. Our findings highlight that while ADL independence is more common in KT candidates than IADL independence, only ADL independence is associated with all three waitlist outcomes. Specifically, unlike IADL independence, ADL independence was associated with lower waitlist mortality and higher rate of $\mathrm{KT}$, mirroring prior research on functional dependence that identified significant reductions in survival among ESKD patients with ADL impairments, but not in ESKD patients with IADL impairments [6]. ADLs represent more basic self-care activities [13], compared with the more complex self-care IADL, which require higher levels of functional ability [15]. It follows, therefore, that overall independence in conducting the most basic, day-to-day selfcare activities has a greater impact on KT waitlist outcomes compared with those activities that require higher levels of functional abilities, particularly among older adults, where ADL independence more than doubled rate of KT. Therefore, clinicians and transplant centers may consider including ADL screening among KT candidates, but not necessarily IADL screening.

Of note, our study found that these associations differed by race and age, such that there was no observed association between ADL independence and waitlist mortality among blacks and no observed association between ADL independence and $\mathrm{KT}$ rate among younger ages. It is possible other protective factors among these subgroups not captured by this study lead to better waitlist outcomes such that the added benefit of being ADL independent is mitigated. The null associations may also be due to the lack of power or unmeasured confounding. Future studies are needed to better understand these causal interactions.

Our study additionally expands on prior findings that demonstrated associations between overall dependence and adverse outcomes in dialysis populations by examining the specific domains of basic and more complex functional independence. We found that independence in eating $(\mathrm{SHR}=0.32,95 \% \mathrm{CI}$ $0.15-0.70 ; \mathrm{P}=0.01)$ and dressing $(\mathrm{SHR}=0.42$, 95\% CI 0.19$0.97 ; \mathrm{P}=0.04)$ were the $\mathrm{ADL}$ domains driving the association with lower waitlist mortality, and independence in walking was driving the association with higher transplant rate $(\mathrm{IRR}=1.79$, 95\% CI 1.19-2.69; $\mathrm{P}=0.005)$. Further investigation into why these domains drive the associations with waitlist outcomes among KT candidates is needed.

There were notable limitations to this study. First, ADLs and IADLs were self-reported, which could have led to potential misclassification. However, self-report is typically how these data are collected in gerontologic studies $[13,15]$. Second, important variables, such as social support, were not collected in this study but may mitigate the impact of ADL and IADL independence on waitlist outcomes. Third, patients were enrolled at the time of evaluation for KT rather than at the time they were referred for dialysis. It is possible that those with the greatest burden of ADL or IADL dependence do not get referred to a transplant center; however, this question is beyond the scope of this research. It is also possible that some patients who were listed were subsequently ineligible for KT due to poor functional status. While this study did not have access to reasons why patients were de-listed, it is unlikely to bias results substantially given that neither ADL independence $(\mathrm{P}=0.504)$ nor IADL independence $(P=0.554)$ were associated with delisting. Additionally, the number of centers incorporated limits inferences to those contexts, and may not be generalizable to the entire US ESKD population being evaluated or listed for KT. However, to our knowledge, this is the largest study to date of ADL and IADL independence, listing practices and outcomes on the KT waitlist. This study has several notable strengths, including its large sample size, its prospective study design, as well as its carefully collected measurements of health characteristics and functional status. Additionally, this study leverages a novel population of ESKD patients who were selected to be healthy enough to withstand transplant surgery.

In conclusion, our results suggest that independence in basic self-care ADL was associated with better $\mathrm{KT}$ access and lower waitlist mortality, underscoring the importance of maintaining functional independence with basic self-care activities. Despite being more common, independence in basic self-care ADLs has a greater impact on KT waitlist outcomes compared with independence in more complex self-care IADLs. Nephrologists, geriatricians and transplant surgeons should consider screening for and identifying the source of dependence in this basic selfcare ADL $[19,33]$, particularly in eating, dressing and walking, but do not need to screen for the more complex self-care IADL. Geriatric syndromes such as frailty are known risk factors of ADL dependence, and may be identified via comprehensive geriatric assessments [19]. Implementing comprehensive geriatric assessments may help reduce the burden and severity of functional dependence by identifying its etiologies and targeting treatment strategies accordingly [19]. Additionally, novel interventions, such as encouraging the use of patient navigators for referrals to prehabilitation to target the identified underlying etiologies of ADL dependence, can then be explored to promote $\mathrm{ADL}$ independence and improve KT waitlist outcomes.

\section{FUNDING}

Funding for this study was provided by the National Institute of Diabetes and Digestive and Kidney Disease and the National Institute of Aging [T32DK007732 to C.E.H., F32AG053025 to C.E.H., K23DK115908 to J.M.G.-W., K24DK101828 to D.L.S., R01AG055781 to M.M.-DeM. and R01DK114074 to M.M.-DeM.].

\section{AUTHORS' CONTRIBUTIONS}

N.M.C. participated in data analysis, interpretation, drafting, critical revision and approval of the article. S.S. participated in 
drafting, critical revision and approval of the article. A.D.M. participated in data analysis and critical revision of the article. C.E.H. participated in critical revision and approval of the article. J.M.G.-W. participated in critical revision and approval of the article. D.C.B. participated in critical revision and approval of the article. S.P.N. participated in data collection, critical revision and approval of the article. D.L.S. participated in concept design, critical revision and approval of the article. M.M.-DeM. participated in concept design, interpretation, securing funding, critical revision and approval of the article.

\section{CONFLICT OF INTEREST STATEMENT}

None declared.

\section{REFERENCES}

1. Kobashigawa J, Dadhania D, Bhorade S et al. Report from the American Society of Transplantation on frailty in solid organ transplantation. Am J Transplant 2019; 19: 984-994

2. McAdams-DeMarco MA, Van Pilsum Rasmussen SE, Chu NM et al. Perceptions and practices regarding frailty in kidney transplantation: results of a national survey. Transplantation 2019 (In press). doi: 10.1097/TP.0000000000002779

3. Ramer SJ, McCall NN, Robinson-Cohen C et al. Health outcome priorities of older adults with advanced CKD and concordance with their nephrology providers' perceptions. J Am Soc Nephrol 2018; 29: 2870-2878

4. Sood MM, Rigatto C, Bueti J et al. The role of functional status in discharge to assisted care facilities and in-hospital death among dialysis patients. Am J Kidney Dis 2011; 58: 804-812

5. Jassal SV, Karaboyas A, Comment LA et al. Functional dependence and mortality in the international dialysis outcomes and practice patterns study (DOPPS). Am J Kidney Dis 2016; 67: 283-292

6. Bossola M, Di Stasio E, Antocicco $\mathrm{M}$ et al. Functional impairment is associated with an increased risk of mortality in patients on chronic hemodialysis. BMC Nephrol 2016; 17: 72

7. McAdams-Demarco MA, Law A, Garonzik-Wang JM et al. Activity of daily living disability and dialysis mortality: better prediction using metrics of aging. J Am Geriatr Soc 2012; 60: 1981-1982

8. Kurella Tamura M, Covinsky KE, Chertow GM et al. Functional status of elderly adults before and after initiation of dialysis. N Engl J Med 2009; 361: 1539-1547

9. Berger JR, Hedayati SS. Renal replacement therapy in the elderly population. Clin J Am Soc Nephrol 2012; 7: 1039

10. Cook WL, Jassal SV. Functional dependencies among the elderly on hemodialysis. Kidney Int 2008; 73: 1289-1295

11. Kavanagh NT, Schiller B, Saxena AB et al. Prevalence and correlates of functional dependence among maintenance dialysis patients. Hemodial Int 2015; 19: 593-600

12. Kutner NG, Zhang R, Allman RM et al. Correlates of ADL difficulty in a large hemodialysis cohort. Hemodial Int 2014; 18: 70-77

13. Katz S, Ford AB, Moskowitz RW et al. Studies of illness in the aged. The index of ADL: a standardized measure of biological and psychosocial function. JAMA 1963; 185: 914-919
14. Goto NA, van Loon IN, Morpey MI et al. Geriatric assessment in elderly patients with end-stage kidney disease. Nephron 2019; 141: 41-48

15. Lawton MP, Brody EM. Assessment of older people: self-maintaining and instrumental activities of daily living. Gerontologist 1969; 9: 179-186

16. Bossola M, Marzetti E, Di Stasio E et al. Prevalence and associated variables of post-dialysis fatigue: results of a prospective multicentre study. Nephrology (Carlton) 2018; 23: 552-558

17. Fried LP, Ferrucci L, Darer J et al. Untangling the concepts of disability, frailty, and comorbidity: implications for improved targeting and care. J Gerontol A Biol Sci Med Sci 2004; 59: M255-M263

18. Painter P, Marcus RL. Assessing physical function and physical activity in patients with CKD. Clin J Am Soc Nephrol 2013; 8: 861-872

19. Hall RK, McAdams-DeMarco MA. Breaking the cycle of functional decline in older dialysis patients. Semin Dial 2018; 31: 462-467

20. Haugen CE, Chu NM, Ying $\mathrm{H}$ et al. Frailty and access to kidney transplantation. Clin J Am Soc Nephrol 2019; 14: 576-582

21. United States Renal Data System. The 2018 Annual Data Report. Chapter 1: Incidence, Prevalence, Patient Characteristics, and Treatment Modalities. http://www.usrds.org/2014/view/ (10 August 2019, date last accessed)

22. Kucirka LM, Grams ME, Balhara KS et al. Disparities in provision of transplant information affect access to kidney transplantation. Am J Transplant 2012; 12: 351-357

23. Saran R, Bragg-Gresham JL, Rayner HC et al. Nonadherence in hemodialysis: associations with mortality, hospitalization, and practice patterns in the DOPPS. Kidney Int 2003; 64: 254-262

24. Rosenthal Asher D, Ver Halen N, Cukor D. Depression and nonadherence predict mortality in hemodialysis treated end-stage renal disease patients. Hemodial Int 2012; 16: 387-393

25. McAdams-DeMarco MA, Law A, Salter ML et al. Frailty as a novel predictor of mortality and hospitalization in individuals of all ages undergoing hemodialysis. J Am Geriatr Soc 2013; 61: 896-901

26. McAdams-DeMarco MA, Suresh S, Law A et al. and falls among adult patients undergoing chronic hemodialysis: a prospective cohort study. $B M C$ Nephrol 2013; 14: 10-18

27. McAdams-DeMarco MA, Tan J, Salter ML et al. Frailty and cognitive function in incident hemodialysis patients. Clin J Am Soc Nephrol 2015; 10: 2181-2189

28. McAdams-DeMarco MA, Ying H, Thomas AG et al. Frailty, inflammatory markers, and waitlist mortality among patients with end-stage renal disease in a prospective cohort study. Transplantation 2018; 102: 1740

29. Chu NM, Deng A, Ying H et al. Dynamic frailty before kidney transplantation-time of measurement matters. Transplantation 103: 1700-1704

30. Charlson M, Szatrowski TP, Peterson J et al. Validation of a combined comorbidity index. J Clin Epidemiol 1994; 47: 1245-1251

31. Hemmelgarn BR, Manns BJ, Quan H, Ghali WA. Adapting the charlson comorbidity index for use in patients with ESRD. Am J Kidney Dis 2003; 42: $125-132$

32. Fine JP, Gray RJ. A Proportional hazards model for the subdistribution of a competing risk. J Am Statistic Assoc 1999; 94: 496-509

33. Hall RK, Haines $\mathrm{C}$, Gorbatkin SM et al. Incorporating geriatric assessment into a nephrology clinic: preliminary data from two models of care. J Am Geriatr Soc 2016; 64: 2154-2158

Received: 2.5.2019; Editorial decision: 1.11.2019 